\title{
Controle sobre GnRH durante o anestro pós-parto em bovinos
}

\author{
GnRH control during bovine postpartum anestrous
}

\author{
João Francisco Coelho de Oliveira ${ }^{I^{*}}$ Paulo Bayard Dias Gonçalves $^{\mathrm{I}}$ Rogério Ferreira $^{\mathrm{I}}$ \\ Bernardo Gasperin ${ }^{I}$ Lucas Carvalho Siqueira ${ }^{\mathrm{I}}$
}

\section{-REVISÃO BIBLIOGRÁFICA-}

\section{RESUMO}

O pós-parto em bovinos é caracterizado como um momento em que as fêmeas bovinas não ovulam, principalmente devido a uma inadequada liberação de gonadotrofinas. Os conceitos e os mecanismos regulatórios do hormônio liberador de gonadotrofinas (GnRH) têm sido descritos isoladamente. Esta revisão aborda a influência da nutrição e amamentação, com enfoque na regulação do GnRH, e fornece conceitos atuais do controle neuroendocrinológico da secreção de GnRH durante o pós-parto em bovinos. Conhecimentos atuais das funções do hormônio inibitório de gonadotrofinas (GnIH), da leptina, dos estrógenos, da kisspeptina e da adiponectina, bem como suas complexas inter-relações durante este periodo estão detalhados para melhor entendimento do assunto.

Palavras-chave: bovinos, GnIH, leptina, kisspeptina, adiponectina .

\section{ABSTRACT}

The bovine postpartum period is characterized as a moment when the ovulation is suppressed, mainly in consequence of insufficient release of gonadotropins. Concepts and regulatory mechanisms of gonadotropin-releasing hormone (GnRH) had been described independently. This review covers the influence of nutrition and suckling with emphasis on GnRH regulation, and provides up to date concepts of neuroendocrine control of GnRH secretion during postpartum in cattle. Current knowledge of gonadotropin-inhibitory hormone $(G n I H)$, leptin, estrogens and kisspeptin during this period are presented in order to provide a better understanding of the subject.

Key words: bovine, GnIH, leptin, kisspeptin, adiponectin.

\section{INTRODUÇÃO}

$\mathrm{O}$ anestro pós-parto em bovinos é um período no qual as fêmeas não ovulam, principalmente devido a uma inadequada liberação de gonadotrofinas. A regulação das funções reprodutivas envolvendo o eixo hipotálamo/hipófise modulado pelos esteróides gonadais é conhecida há bastante tempo. No entanto, especialmente durante o pós-parto, essa regulação constitui-se em um mecanismo de alta complexidade, uma vez que recentemente novos fatores foram descobertos e tiveram suas funções estabelecidas no controle da função reprodutiva.

O GnRH é um decapeptídeo sintetizado por neurônios específicos, localizados na região pré-optica hipotalâmica e liberado de maneira pulsátil na extremidade dos axônios em direção à rede de vasos do sistema porta-hipotalâmico-hipofisário (ESTES et al., 1977). Essa localização privilegiada desses neurônios permite a interpretação de informações centrais e periféricas, conferindo a este neuro-hormônio um papel central na reprodução animal. Sabe-se que a síntese e liberação do GnRH são reguladas por uma complexa associação entre fatores externos e intrínsecos, no qual se destacam, especialmente durante o pós-parto em bovinos, a condição nutricional e a amamentação (RUTTER \& RANDEL, 1984; WILLIAMS et al., 1993). Um dos aspectos que tem

'Laboratório de Biotecnologia e Reprodução Animal, Departamento de Clínica de Grandes Animais, Centro de Ciências Rurais (CCR), Universidade Federal de Santa Maria (UFSM), 97105-900, Santa Maria, RS, Brasil. E-mail: joaofco@biorep.ufsm.br. *Autor para correspondência. 
permanecido misterioso é a forma como o estradiol modula a secreção de GnRH. Nesse sentido, recentemente foi descoberto um hormônio, cujos neurônios secretores apresentam receptores de estrógeno, sendo denominado hormônio inibidor de gonadotrofinas $(\mathrm{GnIH})$. O GnIH desempenha um papel na regulação negativa da secreção de LH em aves (TSUTSUI et al., 2000) e mamíferos (KRIEGSFELD et al., 2006). Esse hormônio é complementar ao papel da kisspeptina, a qual estimula a liberação de GnRH (KAISER \& KUOHUNG, 2005). Um terceiro hormônio, denominado adiponectina, também possui uma função relevante na regulação da hipófise no controle reprodutivo (KADOWAKI \& YAMAUCHI, 2005; RODRIGUEZ-PACHECO et al., 2007).

Os conceitos e os mecanismos regulatórios do GnRH têm sido descritos isoladamente e o entendimento dos mecanismos da influência da nutrição e amamentação durante o pós-parto bovino, com enfoque na regulação do $\mathrm{GnRH}$, teve um progresso considerável na última década. Esta revisão fornece conceitos atuais do controle neuroendocrinológico da secreção de GnRH durante o pós-parto em bovinos. São discutidos os mecanismos centrais e periféricos pelos quais a nutrição e os laços maternais influenciam o processo de síntese e liberação do $\mathrm{GnRH}$ durante o puerpério. Conhecimentos atuais das funções do $\mathrm{GnIH}$, da leptina, dos estrógenos, da kisspeptina e da adiponectina, bem como suas complexas inter-relações durante esse período crítico na vida reprodutiva das fêmeas bovinas serão discutidos para o melhor entendimento do assunto.

Padrão da secreção de gonadotrofinas no pós-parto e a influência da nutrição

A secreção do hormônio folículo estimulante (FSH) em vacas retorna aos padrões do ciclo estral na primeira semana pós-parto (SCHAMS et al., 1978) e, assim, a emergência periódica de ondas de crescimento folicular passa a ser observada (MURPHY et al., 1990). No entanto, o diâmetro máximo atingido pelos folículos dominantes é reduzido no pós-parto precoce e vai aumentando à medida que se aproxima a primeira ovulação. Esse fenômeno fisiológico evidencia que a duração do período anovulatório é determinada por uma frequência inadequada do hormônio luteinizante (LH) para o crescimento final do folículo, ovulação e reinício da ciclicidade.

A produção e a frequência de pulsos do $\mathrm{LH}$ somente são restabelecidas no decorrer do pós-parto em bovinos, sendo esperado que o conteúdo hipofisário desse hormônio esteja normalizado até 30 dias pósparto (RADFORD et al., 1978). No entanto, é importante salientar que vacas que perdem escore corporal no puerpério liberam menos LH após tratamento com $\mathrm{GnRH}$, o que indica que esses animais possuem menores estoques hipofisários desta gonadotrofina (RUTTER \& RANDEL, 1984). Posteriormente, durante o ciclo estral, o controle da síntese e frequência de liberação do LH está sob o controle direto do padrão de liberação do $\mathrm{GnRH}$, o que difere do controle de liberação do FSH, o qual também é regulado pelos níveis de estradiol e inibina (PADMANABHAN \& MCNEILLY, 2001).

A condição nutricional durante o pós-parto precoce em bovinos parece não afetar o tempo necessário para apresentar o primeiro folículo dominante. Porém, o destino deste, refletido pela ovulação ou atresia, é correlacionado com o balanço energético (CICCIOLI et al., 2003). Vacas submetidas a estresse nutricional apresentam redução da liberação de LH (STAGG et al., 1998; CICCIOLI et al., 2003) e, consequentemente, maior intervalo de partos.

A maior necessidade energética na vida das fêmeas bovinas adultas ocorre no pós-parto, quando precisam dispor de energia para manutenção dos processos vitais e produção de leite. Frente à subnutrição, a reprodução é a primeira a ser inibida. $\mathrm{O}$ sistema nervoso central (SNC) interpreta informações periféricas, que o induzem a priorizar a demanda energética para processos indispensáveis. Assim, a síntese e liberação do GnRH é reduzida (I'ANSON et al., 1997; KRIEGSFELD et al., 2006), afetando principalmente a ovulação. Os sítios potenciais de reflexo da nutrição na função ovariana incluem (i) o hipotálamo, via regulação da frequência de GnRH; (ii) a hipófise, regulando a síntese e liberação das gonadotrofinas e hormônio do crescimento; e (iii) o ovário, regulando o crescimento folicular, esteroidogênese e a atividade de fatores de crescimento e suas proteínas de ligação (DISKIN et al., 2003). Nesse conceito de sinalizadores periféricos, a leptina e provavelmente a adiponectina parecem exercer funções centrais na "comunicação" entre nutrição e reprodução.

Leptina: um sinalizador para a reprodução

A leptina é um produto do gene $o b / o b$, que regula o apetite e foi primeiramente estudada em camundongos obesos que possuíam uma mutação nesse gene, apresentando hiperfagia, obesidade e infertilidade (ZHANG et al., 1994). Após a administração exógena de leptina, os animais gradualmente normalizavam seus hábitos alimentares, peso corporal e capacidade reprodutiva (MOUNZIH et al., 1997). Os mesmos sinais clínicos foram observados em humanos que possuem uma mutação no gene que codifica os 
receptores para leptina, impedindo que o hormônio exerça suas funções (BJORBAEK et al., 1998). Devido a essa ação, acredita-se que a leptina exerça influência sobre o eixo reprodutivo, atuando no SNC e em órgãos reprodutivos (SPICER \& FRANCISCO, 1998). A leptina e seus receptores já foram demonstrados em vários tecidos, inclusive no ovário bovino (SARKAR et al, 2010), sendo principalmente produzida pelo tecido adiposo.

A administração exógena de leptina, durante períodos de deficiência nutricional, estimula o eixo hipotalâmico-hipofisário. Isso pode ser evidenciado em novilhas de corte submetidas a jejum, nas quais esse hormônio foi capaz de prevenir a redução na frequência de pulsos de LH e também aumentar a responsividade da hipófise anterior ao GnRH (MACIEL et al., 2004). Já em vacas de corte adultas em jejum, a leptina altera a secreção de GnRH e de LH (AMSTALDEN et al., 2002). Porém, esses efeitos não são observados quando os animais têm suas necessidades nutricionais supridas (MACIEL et al., 2004; AMSTALDEN et al., 2005). Ao contrário do LH, o padrão de secreção de $\mathrm{FSH}$ parece não ser afetado pela subnutrição e pela consequente diminuição na leptinemia, possivelmente devido a uma menor influência do GnRH sobre a produção desse hormônio (PADMANABHAN \& MCNEILLY, 2001).

A secreção de GnRH pode ser influenciada pela leptina, porém o exato mecanismo de interação desses hormônios ainda precisa ser elucidado. Os neurônios produtores de GnRH não possuem receptores para a leptina, por isso acredita-se que sua ação seja mediada via neuropeptideo Y (NPY; MERCER et al., 1996). Esse peptídeo possui um padrão elevado de expressão no hipotálamo e na hipófise anterior de bovinos, sendo encontrado no corpo celular e em terminações nervosas de diversas áreas do cérebro, principalmente naquelas relacionadas com a regulação do consumo alimentar e o balanço energético (THOMAS et al., 1999). A administração central de NPY em ratos induz hiperfagia (STANLEY et al., 1986) e, em bovinos, inibe a liberação de GnRH e LH sem alterar os níveis plasmáticos de FSH (GAZAL et al., 1998). Além disso, a administração central de altas concentrações de NPY em ovelhas bloqueia completamente o pico pré-ovulatório de GnRH (ESTRADA et al., 2003). No entanto, apesar da leptina ser capaz de reduzir a síntese e secreção de NPY (SAHU, 1998), ela não é capaz de atenuar os efeitos sobre a liberação do LH, quando esse neurotransmissor é injetado diretamente no SNC de vacas ovariectomizadas (GARCIA et al., 2004), sugerindo que, em bovinos, a leptina controla apenas as ações pré-secretórias do NPY. Ainda, parece que o NPY é capaz de controlar a liberação do LH na hipófise bovina, pois, após a infusão desse peptídeo diretamente no terceiro ventrículo hipotalâmico, a liberação do LH permaneceu totalmente bloqueada, mesmo após a pulsatilidade do GnRH estar restabelecida (GAZAL et al., 1998). Além de uma regulação na síntese de $\mathrm{GnRH}$, foi demonstrado em camundongos que a leptina altera o potencial de resposta dos gonadotrofos frente ao estímulo do $\mathrm{GnRH}$, através de um aumento da expressão de canais de $\mathrm{Ca}^{++}$ (AVELINO-CRUZ et al., 2009). Esses novos conceitos demonstram mecanismos bastante específicos, pelos quais a leptina é capaz de influenciar a resposta do sistema nervoso central.

Além de atuar no SNC, as ações diretas da leptina nos órgãos reprodutivos parecem ser significativas. A produção da leptina é principalmente extragonadal, pois as variações no líquido folicular acompanham as plasmáticas (SPICER \& FRANCISCO, 1998; GARCIA et al., 2004). Entretanto, as células da teca e granulosa bovina apresentam receptores para essa proteína, sendo a leptina capaz de modular a esteroidogênese (SPICER \& FRANCISCO, 1998; KENDALL et al., 2004; SARKAR et al., 2010).

Influência da amamentação sobre a ciclicidade pósparto

Além da condição corporal, a duração do pós-parto em vacas de corte está relacionada a fatores ambientais e individuais, destacando-se a amamentação. Vacas subnutridas são mais sensíveis aos efeitos inibitórios da amamentação sobre o restabelecimento da ciclicidade (STAGG et al., 1998; CICCIOLI et al., 2003). Além de atuar como fator espoliativo, a presença do bezerro possui ação inibitória sobre a secreção de GnRH/LH, impedindo a ovulação (SHORT et al., 1972; SHORT et al., 1979; WALTERS et al., 1982). A amamentação, quando realizada mais de uma vez ao dia, é capaz de prolongar o período anovulatório do pós-parto (STAGG et al., 1998; LAMB et al., 1999). Já a restrição total da amamentação, por um período mínimo de $48 \mathrm{~h}$, é capaz de aumentar a responsividade da hipófise ao GnRH (TROXEL et al., 1980; SMITH et al., 1983). Isso incrementa principalmente a liberação de $\mathrm{LH}$, aumentando assim a taxa de crescimento folicular e de ovulação e, por consequência, diminui o intervalo de partos (WALTERS et al., 1982).

O efeito negativo da amamentação sobre o restabelecimento da ciclicidade da vaca não ocorre somente pelo ato de sucção do teto, mas também por estímulos táteis, visuais e olfativos, gerados pela presença física do seu bezerro (SILVEIRA et al., 1993; WILLIAMS et al., 1993). Os mecanismos pelos quais 
esses "laços maternais" atuam ainda não são completamente elucidados, porém acredita-se que são resultado de uma complexa interação entre esteróides gonadais, peptídeos opióides endógenos (POE), leptina e possivelmente outros fatores centrais.

O papel dos esteróides no controle do GnRH durante o puerpério

Os estrógenos ovarianos, mesmo em baixos níveis, possuem efeito negativo sobre a frequência de pulsos do GnRH durante o anestro pós-parto (SHORT et al., 1979). Essa resposta do SNC é o oposto da que ocorre em vacas ciclando, em que baixas concentrações de progesterona e a crescente secreção de estrógenos induzem o aumento da frequência de GnRH (SHORT et al., 1979; CHAPPELL \& LEVINE, 2000). O entendimento dos mecanismos neurais pelos quais o estradiol atua nos neurônios secretores de GnRH e sua interação com progesterona, sinalizadores metabólicos e ambientais, parece ser o ponto chave para uma melhor compreensão da fisiologia pós-parto. No entanto, a complexidade das interações hipotalâmicas é uma barreira para o completo entendimento desse mecanismo.

Aparentemente, a principal regulação do GnRH pelo estrógeno se dá de forma indireta, através de grupos de neurônios sensíveis que convergem para região pré-óptica e fazem sinapse com neurônios secretores de GnRH. Em linhagem celular GT1, o estradiol suprime a expressão de GnRH e essa ação é mimetizada pelo uso de agonista ER $\alpha$ (ROY et al., 1999), mas não é antagonizada por antagonista $E R \beta$ (BOWE et al., 2003). Da mesma forma, o uso do fitoestrógeno coumestrol também é capaz de bloquear a expressão gênica de GnRH em linhagem celular GT1, efeito que é suprimido pela adição de antagonista ER $\beta$ (BOWE et al., 2003). Entretanto, não há evidências concretas de uma regulação direta do estradiol sobre os neurônios secretores de GnRH in vivo.

Embora o estradiol cause diretamente uma diminuição na expressão de $\mathrm{GnRH}$, esse efeito pode ser revertido por uma sinalização aferente do estradiol atuando na região periventricular anteroventral (AVPV). Essa região apresenta um dimorfismo sexual bastante acentuado (SIMERLY, 1998), expressão de receptores para estradiol e progesterona (SIMERLY et al., 1996) e recebe a convergência de outras regiões críticas na regulação da frequência de pulsos de GnRH (SIMERLY, 1998). Isso indica que essa região possui um papel chave na interação dos esteróides, sinalizadores metabólicos e ambientais com os neurônios secretores de GnRH. As ações indiretas dos esteróides em ratos são mediadas por neurotransmissores, principalmente o ácido gama aminobutírico (GABA) e o glutamato
(JARRY et al., 1992). Aparentemente, todos os neurônios do GABA localizados no AVPV expressam ERs (PETERSEN et al., 2003). O GABA é um neurotransmissor inibitório abundante no sistema nervoso central com conhecida ação na regulação da expressão e liberação de GnRH (DONOSO et al., 1994). Além disso, o estradiol $17 \beta$ induz a expressão dos receptores de progesterona nos neurônios GABA do AVPV (SHUGHRUE et al., 1997; PETERSEN et al., 2003), o que parece ser indispensável, ao menos em roedores, para que o estradiol induza o pico pré-ovulatório do LH (CHAPPELL \& LEVINE, 2000). A liberação do glutamato também é regulada por estradiol (JARRY et al., 1992) e a ativação dos seus receptores estimula a expressão de GnRH e liberação de LH (DHANDAPANI \& BRANN, 2000). O estradiol atua no controle do GnRH não só alterando as respostas sinápticas via mecanismos genômicos (regulação na expressão de receptores e RNAm), mas também modulando a comunicação entre células de maneira bastante aguda. Essa alteração na resposta celular modifica a responsividade a alguns receptores ligados à proteína G, como opióides, GABA e dopamina (KELLY \& WAGNER, 1999). Cabe ressaltar que a maior parte do conhecimento sobre a atuação da região AVPV no controle do GnRH foi gerado utilizando o roedor como modelo experimental, devido à maior facilidade de manipulação.

Acredita-se que pelo menos parte das ações inibitórias da amamentação seja realizada pelos opióides endógenos (POE), os quais modulariam as ações dos estrógenos no AVPV durante o pós-parto (LESHIN et al., 1991). Os POE, especialmente as $\beta$ endorfinas, atuam no hipotálamo como neurotransmissores, diminuindo a frequência de pulsos do GnRH e, consequentemente de LH. WHISNANT et al. (1986) demonstraram que a inibição dos POE pelo nalaxone, no pós-parto precoce de vacas, determina o restabelecimento na pulsatilidade de LH e ciclicidade. Estudos em ratos sugerem ainda uma ação independente do estrógeno ovariano, sendo que esses neurotransmissores fariam uma interação com hormônios adrenérgicos (dopamina), alterando a secreção de LH. Experimentos em novilhas pré-púberes demonstraram os efeitos isolados dos POE e de neurotransmissores adrenérgicos, porém não confirmaram um efeito cumulativo entre eles (HONARAMOOZ et al., 2000). Em um trabalho utilizando ratas em lactação, foi observado que a habilidade do estradiol em ativar os neurônios do AVPV e do GnRH (marcado pela imunoreatividade de c-Fos) aumenta após o pico da lactação (ABIZAID et al., 2004). Além disso, os mesmos autores demonstraram que, em 
ratas com restrição na dieta, mesmo após o pico da lactação, há uma diminuição na capacidade do estradiol em induzir imunoreatividade de c-Fos no AVPV. Já aquelas alimentadas ad libitum, a imunoreatividade de c-Fos após o pico da lactação foi similar aos animais não lactantes (ABIZAID et al., 2004). Esses resultados sugerem que o efeito da dieta e da lactação na capacidade do estradiol em aumentar a síntese de $\mathrm{GnRH}$ é mediado, pelo menos em parte, pelo AVPV. Ainda, a inibição da liberação de LH durante a amamentação possui mecanismos de controle independentes dos esteróides gonadais, pois vacas castradas amamentando apresentam redução na secreção dessa gonadotrofina, em relação a vacas desmamadas (GARCIA-WINDER et al., 1984).

Controle gonadotrófico mediado pela Kisspeptina, GnIH e Adiponectina

A kisspeptina atua via receptor GPR54 e parece estabelecer uma ligação direta entre sinalizadores periféricos, como, por exemplo, esteróides gonadais e leptina, e a síntese de GnRH (GOTTSCH et al., 2004; SMITH et al., 2005; BACKHOLER et al., 2010). Atuando como um potente modulador do GnRH, é possível verificar a ação da kisspeptina em diversas espécies, após administração central ou periférica (GOTTSCH et al., 2004). A ação dessa proteína no eixo gonadotrófico parece ser iniciada em regiões hipotalâmicas distintas. Em roedores, existem duas regiões que expressam o gene Kiss-1: a dos núcleos arqueados (ARQ) e a AVPV. É interessante ressaltar que, nessas espécies, os esteróides diminuem a expressão de Kiss-1 no grupo de neurônios ARQ, sendo observado um efeito oposto na região AVPV (SMITH et al., 2005). Nessa última, o processo de gonadectomia é capaz de reduzir a expressão de RNAm para Kiss-1, porém esse efeito é revertido pelo tratamento com estrógenos (SMITH et al., 2005). Esses resultados sugerem que, em roedores, os neurônios da AVPV são os principais responsáveis pelo feedback positivo dos esteróides na síntese de $\mathrm{GnRH}$, enquanto os ARQ pelo feedback negativo. No entanto, no hipotálamo de ruminantes, os neurônios do ARQ parecem acumular funções, "traduzindo" tanto os sinais periféricos estimulatórios, quanto os inibitórios para a secreção de kisspeptinas e, por consequência, de GnRH (ESTRADA et al., 2006; FRANCESCHINI et al., 2006; SMITH, 2009).

Além dos esteróides, a leptina parece também utilizar a rota da kisspeptina para controlar o eixo gonadotrófico. Nos neurônios em que se observa transcrição de RNAm para Kiss-1 também ocorre expressão de receptores para leptina. Camundongos portadores de mutação inativadora no gene da leptina apresentam diminuição na expressão de Kiss-1 na região ARQ e, após o tratamento com leptina, elevam os níveis de expressão (LUQUE et al., 2007). Esses resultados sugerem que a kisspeptina pode ser responsável pelo menos por parte dos efeitos da leptina na reprodução.

As funções do sistema Kiss-1/GPR54 durante o puerpério também estão sendo evidenciadas, pois aparentemente ratas nessa fase apresentam menor sensibilidade à administração da kisspeptina (YAMADA et al., 2007). Além disso, quando se sobrepõem os efeitos da amamentação e nutrição, determinando a diminuição do padrão de secreção do $\mathrm{GnRH}$, a transcrição de RNAm de Kiss-1 está diminuída no ARQ, mesmo em ratas ovariectomizadas (OHKURA et al., 2009) No entanto, o padrão de expressão de receptores GPR54 nos núcleos hipotalâmicos ainda necessita ser melhor elucidado.

$\mathrm{O} \mathrm{GnIH}$ e a adiponectina foram descobertos recentemente e já são apontados como fundamentais na regulação neuroendócrina da reprodução. $\mathrm{O} \mathrm{GnIH}$ foi inicialmente isolado do hipotálamo de codornas, tendo sido demonstrado que ele inibe a secreção do LH da hipófise anterior in vivo e in vitro (TSUTSUI et al., 2000). O gene que codifica o precursor da proteína para GnIH já foi clonado em várias espécies de aves e possui um alto grau de homologia entre espécies (SATAKE et al., 2001; IKEMOTO \& PARK, 2005). Nesses animais, neurônios imunoreativos (ir) para o GnIH são exclusivamente encontrados no núcleo hipotalâmico paraventricular, enquanto fibras GnIH são encontradas em outras regiões do cérebro, incluindo a eminência média (UKENA et al., 2003). Neurônios GnIHir possuem estreita relação com células GnRH-ir, sugerindo uma possível interação entre neurônios GnIH e GnRH (KRIEGSFELD et al., 2006).

A administração de GnIH tanto por via periférica como intracerebroventricular em hamsters ovariectomizadas resultou na redução das concentrações de LH plasmático, proporcionando evidências do papel do GnIH como um fator inibidor de gonadotrofinas (KRIEGSFELD et al., 2006). cDNAs que codificam novos RFRPs similares ao GnIH têm sido descritos (HINUMA et al., 2000). Os transcritos de humanos e bovinos codificam três peptídeos denominados RFRP-1, -2, e -3. Especificamente em bovinos, foi demonstrado que o RFRP-3 suprime in vitro a secreção de LH em cultivo de células de hipófise, bem como a frequência de pulsos de LH em machos castrados (KADOKAWA et al., 2009).

A adiponectina é uma proteína semelhante ao colágeno que pertence à família das adipocinas, as quais exercem um papel importante na regulação da ingestão de alimentos, homeostase de energia e 
imunidade (TRAYHURN, 2005). A adiponectina possui dois tipos de receptores: AdipoR1 e AdipoR2. Eles apresentam homologia de $67 \%$, possuindo 7 domínios transmembrana e estão presentes em vários tecidos, incluindo hipotálamo, adipócitos e placenta (KADOWAKI \& YAMAUCHI, 2005). A adiponectina também regula a secreção e expressão em dois tipos de células da hipófise de ratos, os somatotrófos e os gonadotrófos. A expressão de receptores AdipoR1 e AdipoR2 na hipófise sugere um sistema regulatório local da adiponectina em nível de hipófise (RODRIGUEZ-PACHECO et al., 2007). Evidências, indicando o envolvimento da adiponectina no controle dos fenômenos reprodutivos em bovinos, incluem a presença de RNAm para seus receptores em células da teca e granulosa em folículos antrais de diferentes estádios de desenvolvimento.

Esses achados, avaliados em conjunto, suportam a existência de rotas paralelas e independentes da clássica ação dos esteróides no controle de secreção do GnRH em animais no pós-parto, em que o $\mathrm{GnIH}$, a kisspeptina e a adiponectina desempenham funções na regulação das gonadotrofinas e, consequentemente, da atividade ovariana. No entanto, as informações sobre o papel do GnIH e da adiponectina ainda são bastante restritas em bovinos.

\section{CONCLUSÃO}

Ainda existem várias lacunas a serem preenchidas para o completo entendimento da ação dos laços maternais e o controle da reprodução. A complexa interação entre nutrição, amamentação e retorno à ciclicidade precisa ser melhor investigada. As pesquisas têm avaliado a importância de cada fator isoladamente, porém, compreender a interação entre os fatores é imprescindível. Assim, mais estudos precisam ser realizados, gerando novos conhecimentos e novas tecnologias para o incremento da produtividade dos rebanhos bovinos.

\section{REFERÊNCIAS}

ABIZAID, A. et al. Effects of estradiol on immediate early gene expression associated with ovulation in lactating rats: role of nutritional status. Brain Research, v.1001, n.1-2, p.7277, 2004. Disponível em: <http://www.sciencedirect.com/ $\mathrm{sc}$ i e n c e/a r i c 1 e/ B 6 S Y R - 4 B G 44 X H - 2/1/ aab58c7fb078b986cd14795863fec24d>. Acesso em: 14 set. 2010. doi:10.1016/j.brainres.2003.10.069.

AMSTALDEN, M. et al. Central infusion of recombinant ovine leptin normalizes plasma insulin and stimulates a novel hypersecretion of luteinizing hormone after short-term fasting in mature beef cows. Biology Reproduction, v.66, n.5, p.1555-1561, 2002. Disponível em: <http://www.biolreprod.org/ content/66/5/1555.long>. Acesso em: 14 set. 2010. doi: 10.1095/ ?biolreprod66.5.1555.

AMSTALDEN, M. et al. Effects of leptin on gonadotropin-releasing hormone release from hypothalamic-infundibular explants and gonadotropin release from adenohypophyseal primary cell cultures: further evidence that fully nourished cattle are resistant to leptin. Animal Reproduction Science, v.85, n.1-2, p.41-52, 2005. Disponível em: <http://www.sciencedirect.com/science/article/ B6T43-4CFV6HJ-2/2/255f99340cfd3586bb990d7ee0b5e88f >. Acesso em: 14 set. 2010. doi: 10.1095/?biolreprod66.5.1555.

AVELINO-CRUZ, J.E. et al. Leptin increases L-type Ca2+ channel expression and GnRH-stimulated LH release in L[beta]T2 gonadotropes. Molecular and Cellular Endocrinology, v.298, n.1-2, p.57-65, 2009. Disponível em: $<$ http://www.sciencedirect.com/science/article/B6T3G4TDVMJJ-1/2/548266acaf50143fecd05bea2b6d9376>. Acesso em: 14 set. 2010. doi:10.1016/j.mce.2008.09.003.

BACKHOLER, K. et al. Kisspeptin cells in the ewe brain respond to leptin and communicate with neuropeptide $\mathrm{Y}$ and proopiomelanocortin cells. Endocrinology, v.151, n.5, p.22332243, 2010. Disponível em: <http://endo.endojournals.org/cgi/ content/abstract/151/5/2233>. Acesso em: 14 set. 2010. doi:10.1210/en.2009-1190.

BJORBAEK, C. et al. Expression of leptin receptor isoforms in rat brain microvessels . Endocrinology, v.139, n.8, p.34853491, 1998. Disponível em: <http://endo.endojournals.org/ cgi/content/full/139/8/3485>. Acesso em: 14 set. 2010 . doi: 10.1210/en.139.8.3485.

BOWE, J. et al. The effects of the phytoestrogen, coumestrol, on gonadotropin-releasing hormone $(\mathrm{GnRH}) \mathrm{mRNA}$ expression in GT1-7 GnRH neurones. Journal of Neuroendocrinology, v.15, n.2, p.105-108, 2003. Disponível em: <http://www.blackwellsynergy.com/doi/abs/10.1046/j.1365-2826.2003.00991.x>. Acesso em: 14 set. 2010. doi:10.1046/j.1365-2826.2003.00991.x.

CHAPPELL, P.E.; LEVINE, J.E. Stimulation of gonadotropinreleasing hormone surges by estrogen. I. Role of hypothalamic progesterone receptors. Endocrinology, v.141, n.4, p.14771485, 2000. Disponível em: <http://endo.endojournals.org/ cgi/content/abstract/141/4/1477>. Acesso em: 14 set. 2010. doi:10.1210/en.141.4.1477.

CICCIOLI, N.H. et al. Influence of body condition at calving and postpartum nutrition on endocrine function and reproductive performance of primiparous beef cows. Journal of Animal Science, v.81, n.12, p.3107-3120, 2003. Disponível em: <http:/ /www.ncbi.nlm.nih.gov/entrez/query.fcgi? $\mathrm{cmd}=$ Retrieve $\& \mathrm{db}=$ PubMed\&dopt $=$ Citation\&list_uids $=14677867>$. Acesso em: 14 set. 2010.

DHANDAPANI, K.M.; BRANN, D.W. The role of glutamate and nitric oxide in the reproductive neuroendocrine system. Biochemistry and Cell Biology, v.78, n.3, p.165-179, 2000. Disponível em: $<$ https://article.pubs.nrc-cnrc.gc.ca/RPAS/rpv?issn=0829$8211 \&$ volume $=78 \&$ issue $=3 \&$ startPage $=165 \&$ sepr $=v t>$. Acesso em: 14 set. 2010 . doi: 10.1139/bcb-78-3-165.

DISKIN, M.G. et al. Effects of nutrition and metabolic status on circulating hormones and ovarian follicle development in cattle. Animal Reproduction Science, v.78, n.3-4, p.345- 
370, 2003. Disponível em: <http://www.sciencedirect.com/ $\mathrm{science} / \mathrm{artic} 1 \mathrm{e} / \mathrm{B} 6 \mathrm{~T} 43-48 \mathrm{R} 7 \mathrm{CD} \mathrm{B}-4 / 1 /$ 50e59d5d1b920b32c50cafb42224e2c1>. Acesso em: 14 set. 2010. doi:10.1016/S0378-4320(03)00099-X.

DONOSO, A.O. et al. Regulation of luteinizing hormonereleasing hormone and luteinizing hormone secretion by hypothalamic amino acids. Brazilian Journal of Medical and Biological Research, v.27, n.4, p.921-932, 1994. Disponível em: <http://www.ncbi.nlm.nih.gov/pubmed/ 8087094>. Acesso em: 14 set. 2010.

ESTES, K.S. et al. Localization of gonadotropin releasing hormone $(\mathrm{GnRH})$ within the bovine hypothalamus. Biology of Reproduction, v.17, n.5, p.706-711, 1977. Disponível em: <http://www.biolreprod.org/content/17/5/706.abstract>. Acesso em: 14 set. 2010. doi 10.1095/biolreprod17.5.706.

ESTRADA, K.M. et al. Elevated kiss-1 expression in the arcuate nucleus prior to the cyclic preovulatory gonadotrophin-releasing hormone/lutenising hormone surge in the ewe suggests a stimulatory role for kisspeptin in oestrogen-positive feedback. Journal of Neuroendocrinology, v.18, n.10, p.806-809, 2006. Disponível em: $<$ http://onlinelibrary.wiley.com/doi/ 10.1111/j.1365-2826.2006.01485.x/pdf>. Acesso em: 14 set. 2010. doi: $10.1111 /$ j.1365-2826.2006.01485.x.

ESTRADA, K.M. et al. Neuropeptide Y (NPY) delays the oestrogen-induced luteinizing hormone (LH) surge in the ovariectomized ewe: further evidence that NPY has a predominant negative effect on LH secretion in the ewe. Journal of Neuroendocrinology, v. 15, n.11, p.1011-1020, 2003. Disponível em: <http://onlinelibrary.wiley.com/doi/ 10.1046/j.1365-2826.2003.01087.x/pdf>. Acesso em: 14 set. 2010. doi: $10.1046 / j .1365-2826.2003 .01087 . x$.

FRANCESCHINI, I. et al. Kisspeptin immunoreactive cells of the ovine preoptic area and arcuate nucleus co-express estrogen receptor alpha. Neuroscience Letters, v.401, n.3, p.225230, 2006. Disponível em: <http://www.sciencedirect.com/ $\mathrm{sc}$ i e n c e/a r t i c 1 e/B 6 T 0 G - 4 J R V D $9 \mathrm{~N}-1 / 2$ / c1371cc37ab839830dccdd9c6b424bbd $>$. Acesso em: 14 set. 2010. doi:10.1016/j.neulet.2006.03.039.

GARCIA, M.R. et al. Leptin attenuates the acute effects of centrally administered neuropeptide $\mathrm{Y}$ on somatotropin but not gonadotropin secretion in ovariectomized cows. Domestic Animal Endocrinology, v.26, n.3, p.189-200, 2004. Disponível em: <http:/ /www.sciencedirect.com/science?_ob=ArticleURL\&_udi=B6T62$4 \mathrm{~B} 6 \mathrm{~K} 8 \mathrm{R} 9-2 \&$ user $=687358 \&$ coverDate $=04 \% 2 \mathrm{~F} 30 \% 2 \mathrm{~F} 2004 \&$ rdoc $=1 \& \_$fmt $=$high\&_orig $=$search \&_origin $=$search\&_sort $=\mathrm{d} \& \_$docan chor $=\&$ view $=$ c\& acct $=$ C000037899\& version $=1 \&$ urlVersion $=0$ \& us e r i d $=687358 \&$ m d $5=\mathrm{c} 5$ f 9 a 9 f 4 e e 075427 9dc49951b0b4a051\&searchtype $=\mathrm{a}>$. Acesso em: 14 set. 2010. doi:10.1016/j.domaniend.2003.10.003.

GARCIA-WINDER, M. et al. Effect of suckling and ovariectomy on the control of luteinizing hormone secretion during the postpartum period in beef cows. Biology of Reproduction, v.31, n.4, p.771-778, 1984. Disponível em: <http:// www.biolreprod.org/content/31/4/771.long $>$. Acesso em: 14 set. 2010. doi: 10.1095/?biolreprod31.4.771.

GAZAL, O.S. et al. Gonadotropin-releasing hormone secretion into third-ventricle cerebrospinal fluid of cattle: correspondence with the tonic and surge release of luteinizing hormone and its tonic inhibition by suckling and neuropeptide Y. Biology of Reproduction, v.59, n.3, p.676-683, 1998. Disponível em: $<$ http://www.biolreprod.org/content/59/3/676.full.pdf $>$. Acesso em: 14 set. 2010. doi: 10.1095/?biolreprod59.3.676.

GOTTSCH, M.L. et al. A role for kisspeptins in the regulation of gonadotropin secretion in the mouse. Endocrinology, v.145, n.9, p.4073-4077, 2004. Disponível em: <http:// endo.endojournals.org/cgi/content/full/145/9/4073>. Acesso em: 14 set. 2010 . doi:10.1210/en.2004-0431.

HINUMA, S. et al. New neuropeptides containing carboxyterminal RFamide and their receptor in mammals. Nature Cell Biology, v.2, n.10, p.703-708, 2000. Disponível em: $<$ http://www.nature.com/doifinder/10.1038/35036326 >. Acesso em: 14 set. 2010. doi:10.1038/35036326.

HONARAMOOZ, A. et al. Opioidergic, dopaminergic and adrenergic regulation of $\mathrm{LH}$ secretion in prepubertal heifers. Journal of Reproduction and Fertility, v.119, n.2, p.207215, 2000. Disponível em: <http://www.reproductiononline.org/cgi/reprint/119/2/207>. Acesso em: 14 set. 2010.

I'ANSON, H. et al. Regional differences in the distribution of gonadotropin-releasing hormone cells between rapidly growing and growth-restricted prepubertal female sheep. Endocrinology, v.138, n.1, p.230-236, 1997. Disponível em: $<$ http://endo.endojournals.org/cgi/reprint/138/1/230 > . Acesso em: 14 set. 2010 . doi: 10.1210/en.138.1.230.

IKEMOTO, T.; PARK, M.K. Chicken RFamide-related peptide (GnIH) and two distinct receptor subtypes: identification, molecular characterization, and evolutionary considerations. Journal of Reproduction and Development, v.51, n.3, p.359-377, 2005. Disponível em: $<$ http://www.jstage.jst.go.jp/ article/jrd/51/3/51_359/_article>. Acesso em: 14 set. 2010. doi:10.1262/jrd.16087.

JARRY, H. et al. Amino acid neurotransmitter release in the preoptic area of rats during the positive feedback actions of estradiol on LH release. Neuroendocrinology, v.56, n.2, p.133-140, 1992. Disponível em: Acesso em: 14 set. 2010. KADOKAWA, H. et al. Bovine C-terminal octapeptide of RFamide-related peptide-3 suppresses luteinizing hormone (LH) secretion from the pituitary as well as pulsatile LH secretion in bovines. Domestic Animal Endocrinology, v.36, n.4, p.219224, 2009. Disponível em: <http://www.ncbi.nlm.nih.gov/ e n tre z / query. f c g i ? c m d = Retrieve \& d b $=$ P u b Med\&dopt=Citation\&list_uids=19328642>. Acesso em: 14 set. 2010. doi:10.1016/j.domaniend.2009.02.001.

KADOWAKI, T.; YAMAUCHI, T. Adiponectin and adiponectin receptors. Endocrine Reviews, v.26, n.3, p.439451, 2005. Disponível em: <http://edrv.endojournals.org/cgi/ reprint/26/3/439>. Acesso em: 14 set. 2010. doi: 10.1210/ er.2005-0005.

KAISER, U.B.; KUOHUNG, W. KiSS-1 and GPR54 as new players in gonadotropin regulation and puberty. Endocrine, v.26, n.3, p.277-284, 2005. Disponível em: <http:// www.springerlink.com/content $/ 41414812056356 \mathrm{~h} 1 />$. Acesso em: 14 set. 2010 . doi: 10.1385/ENDO:26:3:277. 
KELLY, M.J.; WAGNER, E.J. Estrogen modulation of g-proteincoupled receptors. Trends in Endocrinology and Metabolism, v.10, n.9, p.369-374, 1999. Disponível em: <http:/ /www.sciencedirect.com/science/article/B6T3K-3XM8NXP-4/1/ 0e0f67785abceb2dc140e2f4ce84877f $>$. Acesso em: 14 set. 2010. doi:10.1016/S1043-2760(99)00190-3.

KRIEGSFELD, L.J. et al. Identification and characterization of a gonadotropin-inhibitory system in the brains of mammals. Proceedings of the National Academy Sciences, v.103, n.7, p.2410-2415, 2006. Disponível em: <http://www.pnas.org/ content/103/7/2410.long $>$. Acesso em: 14 set. 2010 . doi: 10.1073/pnas.0511003103.

LAMB, G.C. et al. Twice daily suckling but not milking with calf presence prolongs postpartum anovulation. Journal of Animal Science, v.77, n.8, p.2207-2218, 1999. Disponível em: <http://jas.fass.org/cgi/reprint/77/8/2207 >. Acesso em: 14 set. 2010 .

LESHIN, L.S. et al. The bovine preoptic area and median eminence: sites of opioid inhibition of luteinizing hormonereleasing hormone secretion. Journal of Animal Science, v.69, n.9, p.3733-3746, 1991. Disponível em: <http:// jas.fass.org/cgi/reprint/69/9/3733 >. Acesso em: 14 set. 2010 .

LUQUE, R.M. et al. Regulation of hypothalamic expression of KiSS-1 and GPR54 Genes by metabolic factors: analyses using mouse models and a cell line. Endocrinology, v.148, n.10, p.4601-4611, 2007. Disponível em: <http:// endo.endojournals.org/cgi/content/abstract/148/10/4601>. Acesso em: 14 set. 2010. doi: 10.1210/en.2007-0500.

MACIEL, M.N. et al. Chronic administration of recombinant ovine leptin in growing beef heifers: effects on secretion of LH, metabolic hormones, and timing of puberty. Journal of Animal Science, v.82, n.10, p.2930-2936, 2004. Disponível em: <http:/ /www.ncbi.nlm.nih.gov/entrez/query.fcgi? $\mathrm{cmd}=$ Retrieve \&db=PubM ed\&dopt $=$ Citation\&list_uids $=15484944>$. Acesso em: 14 set. 2010 .

MERCER, J.G. et al. Coexpression of leptin receptor and preproneuropeptide $\mathrm{Y}$ mRNA in arcuate nucleus of mouse hypothalamus. Journal of Neuroendocrinology, v.8, n.10, p.733-735, 1996. Disponível em: <http://www.ncbi.nlm.nih.gov/ en trez/query.f c g i ? c m d = Retri e ve \& d b $=P$ u bMed\&dopt $=$ Citation\&list_uids $=8910801>$. Acesso em: 14 set. 2010. doi: 10.1046/j.1365-2826.1996.05161.x.

MOUNZIH, K. et al. Leptin treatment rescues the sterility of genetically obese ob/ob males. Endocrinology, v.138, n.3, p.11901193, 1997. Disponível em: <http://endo.endojournals.org/cgi/ content/full/138/3/1190 >. Acesso em: 14 set. 2010. doi: 10.1210/ en.138.3.1190.

MURPHY, M.G. et al. Pattern of follicular growth and resumption of ovarian activity in post-partum beef suckler cows. Journal of Reproduction and Fertility, v.90, n.2, p.523-533, 1990. Disponível em: <http://www.reproductiononline.org/cgi/content/abstract/90/2/523>. Acesso em: 14 set. 2010. doi:10.1530/jrf.0.0900523.

OHKURA, S. et al. Physiological role of metastin/kisspeptin in regulating gonadotropin-releasing hormone $(\mathrm{GnRH})$ secretion in female rats. Peptides, v.30, n.1, p.49-56, 2009. Disponível em: <http://www.sciencedirect.com/science/article/B6T0M4T7XGJD-3/2/d32f41c72b106a0f12280e883c86e1 fa>. Acesso em: 14 set. 2010. doi:10.1016/j.peptides.2008.08.004.
PADMANABHAN, V.; MCNEILLY, A. Is there an FSHreleasing factor? Reproduction, v.121, n.1, p.21-30, 2001. Disponível em: <http://www.reproduction-online.org/cgi/ content/abstract/121/1/21>. Acesso em: 14 set. 2010. doi: $10.1530 /$ rep. 0.1210021 .

PETERSEN, S.L. et al. Direct and indirect regulation of gonadotropin-releasing hormone neurons by estradiol. Biology of Reproduction, v.69, n.6, p.1771-1778, 2003. Disponível em: $<$ http://www.biolreprod.org/content/69/6/1771.full.pdf + html $>$. Acesso em: 14 set. 2010. doi: 10.1095/?biolreprod.103.019745.

RADFORD, H.M. et al. Ovarian function in suckling and nonsuckling beef cows post partum. Journal of Reproduction and Fertility, v.54, n.1, p.49-56, 1978. Disponível em: <http:/ /www.reproduction-online.org/cgi/content/abstract/54/1/49>. Acesso em: 14 set. 2010. doi:10.1530/jrf.0.0540049.

RODRIGUEZ-PACHECO, F. et al. Regulation of pituitary cell function by adiponectin. Endocrinology, v.148, n.1, p.401410, 2007. Disponível em: <http://endo.endojournals.org/cgi/ reprint/148/1/401.pdf>. Acesso em: 14 set. 2010. doi: 10.1210/ en.2006-1019.

ROY, D. et al. Estrogen directly represses gonadotropin-releasing hormone $(\mathrm{GnRH})$ gene expression in estrogen receptor- $\alpha(\mathrm{ER} \alpha)$ and ER $\beta$ expressing GT1-7 GnRH neurons. Endocrinology, v.140, n.11, p.5045-5053, 1999. Disponível em: $<\mathrm{http}: / /$ endo.endojournals.org/cgi/content/abstract/140/11/5045>. Acesso em: 14 set. 2010. doi:10.1210/en.140.11.5045.

RUTTER, L.M.; RANDEL, R.D. Postpartum nutrient intake and body condition: effect on pituitary function and onset of estrus in beef cattle. Journal of Animal Science, v.58, n.2, p.265-274, 1984. Disponível em: <http://jas.fass.org/cgi/ content/abstract/58/2/265>. Acesso em: 14 set. 2010.

SAHU, A. Leptin decreases food intake induced by melaninconcentrating hormone $(\mathrm{MCH})$, galanin $(\mathrm{GAL})$ and neuropeptide $\mathrm{Y}(\mathrm{NPY})$ in the rat. Endocrinology, v.139, n.11, p.47394742, 1998. Disponível em: <http://www.ncbi.nlm.nih.gov/ entrez/query.f c gi ? c m d = Retrieve \& d b = P ub M ed\&dopt $=$ Citation\&list_uids $=9794487>$. Acesso em: 14 set. 2010. doi: 10.1210/en.139.11.4739.

SATAKE, H. et al. Characterization of a cDNA encoding a novel avian hypothalamic neuropeptide exerting an inhibitory effect on gonadotropin release. Biochemical Journal, v.354, n.2, p.379-385, 2001. Disponível em: <http://www.ncbi.nlm.nih.gov/ entrez/query.f g i ? c m d = Retrieve \& d b = P u b Med\&dopt $=$ Citation\&list_uids $=11171117>$. Acesso em: 14 set. 2010 .

SCHAMS, D. et al. Profiles of LH, FSH and progesterone in postpartum dairy cows and their relationship to the commencement of cyclic functions. Theriogenology, v.10, n.6, p.453-468, 1978. Disponível em: <http://www.sciencedirect.com/science/article/B6TCM49NH8M3-9Y/2/88b66951e3b51a5ad5eb07dd7a8c3190>. Acesso em: 14 set. 2010. doi:10.1016/0093-691X(78)90135-8.

SHORT, R.E. et al. Effects of suckling and mastectomy on bovine postpartum reproduction. Journal of Animal Science, v.34, n.1, p.70-74, 1972. Disponível em: <http://jas.fass.org/ cgi/content/abstract/34/1/70>. Acesso em: 14 set. 2010.

SHORT, R.E. et al. Factors affecting estrogen-induced LH release in the cow. Biology of Reproduction, v.21, n.3, p.683-689, 1979. Disponível em: <http://www.ncbi.nlm.nih.gov/entrez/ qu e r y.f c g i ? c m d $=R$ e t r i e v e \& d b $=P$ u b Med\&dopt $=$ Citation\&list_uids $=497325>$. Acesso em: 14 set. 2010. doi: 10.1095/?biolreprod21.3.683. 
SHUGHRUE, P.J. et al. Regulation of progesterone receptor messenger ribonucleic acid in the rat medial preoptic nucleus by estrogenic and antiestrogenic compounds: an in situ hybridization study. Endocrinology, v.138, n.12, p.5476-5484, 1997. Disponível em: <http://endo.endojournals.org/cgi/ content/abstract/138/12/5476>. Acesso em: 14 set. 2010. doi:10.1210/en.138.12.5476.

SILVEIRA, P.A. et al. Evidence for maternal behavior as a requisite link in suckling-mediated anovulation in cows. Biology of Reproduction, v.49, n.6, p.1338-1346, 1993. Disponível em: <http:/ /www.ncbi.nlm.nih.gov/entrez/query.fcgi?cmd=Retrieve\&db=PubM ed\&dopt=Citation\&list_uids=8286616>. Acesso em: 14 set. 2010. doi: 10.1095/?biolreprod49.6.1338

SIMERLY, R.B. Organization and regulation of sexually dimorphic neuroendocrine pathways. Behavioural Brain Research, v.92, n.2, p.195-203, 1998. Disponível em: <http:/ /www.sciencedirect.com/science/article/B6SYP-3TCVYMH-B/ $1 /$ fe80506505734906828ef7841886a225>. Acesso em: 14 set. 2010. doi:10.1016/S0166-4328(97)00191-5.

SIMERLY, R.B. et al. Ovarian steroid regulation of estrogen and progesterone receptor messenger ribonucleic acid in the anteroventral periventricular nucleus of the rat. Journal of Neuroendocrinology, v.8, n.1, p.45-56, 1996.

SMITH, J.T. Sex steroid control of hypothalamic Kiss 1 expression in sheep and rodents: comparative aspects. Peptides, v.30, n.1, p.94-102, 2009. Disponível em: <http:/ /www.sciencedirect.com/science/article/B6T0M-4T9CCN3-1/2/ 4cab8e96bae85aa43b1a480a80a293e5>. Acesso em: 14 set. 2010. doi:10.1016/j.peptides.2008.08.013.

SMITH, J.T. et al. Differential regulation of kiss-1 mRNA expression by sex steroids in the brain of the male mouse. Endocrinology, v.146, n.7, p.2976-2984, 2005. Disponível em: <http://endo.endojournals.org/cgi/content/abstract/146/7/ 2976>. Acesso em: 14 set. 2010. doi:10.1210/en.2005-0323.

SMITH, M.F. et al. Pituitary and ovarian responses to gonadotropin releasing hormone, calf removal and progestogen in anestrous beef cows. Journal of Animal Science, v. 57, n.2, p.418-424, 1983. Disponível em: <http://jas.fass.org/cgi/ content/abstract/57/2/418>. Acesso em: 14 set. 2010.

SPICER, L.J.; FRANCISCO, C.C. Adipose obese gene product, leptin, inhibits bovine ovarian thecal cell steroidogenesis. Biology of Reproduction, v.58, n.1, p.207-212, 1998. Disponível em: <http:// www.ncbi.nlm.nih.gov/entrez/query.fcgi? $\mathrm{cmd}=$ Retrieve $\& \mathrm{db}=$ PubMed\& dopt $=$ Citation\&list_uids $=9472942>$. Acesso em: 14 set. 2010. doi:10.1095/?biolreprod58.1.207.

STAGG, K. et al. Effect of calf isolation on follicular wave dynamics, gonadotropin and metabolic hormone changes, and interval to first ovulation in beef cows fed either of two energy levels postpartum. Biology of Reproduction, v.59, n.4, p.777783, 1998. Disponível em: <http://www.ncbi.nlm.nih.gov/ en tre z/ query.f c g i ? c md = Retrieve \& d b $=P$ ub Med\&dopt $=$ Citation\&list_uids $=9746725>$. Acesso em: 14 set. 2010. doi: $10.1095 /$ ?biolreprod59.4.777.

THOMAS, M.G. et al. Injection of neuropeptide $\mathrm{Y}$ into the third cerebroventricle differentially influences pituitary secretion of luteinizing hormone and growth hormone in ovariectomized cows. Domestic Animal Endocrinology, v.16, n.3, p.159-169, 1999. Disponível em: <http:// www.ncbi.nlm.nih.gov/entrez/query.fcgi? $\mathrm{cmd}=$ Retrieve $\& \mathrm{db}=\mathrm{Pub}$
Med\&dopt $=$ Citation\&list_uids $=10343918>$. Acesso em: 14 set. 2010. doi:10.1016/S0739-7240(99)00009-0.

TRAYHURN, P. Endocrine and signalling role of adipose tissue: new perspectives on fat. Acta Physiologica Scandinavica, v.184, n.4, p.285-293, 2005. Disponível em: <http:// on linelibrary.wiley.com/doi/ $10.1111 /$ j . 1365 201X.2005.01468.x/pdf>. Acesso em: 14 set. 2010. doi: 10.1111/j.1365-201X.2005.01468.x.

TROXEL, T.R. et al. Ovulation and reproductive hormones following steroid pretreatment, calf removal and GnRH in postpartum suckled beef cows. Journal of Animal Science, v.51, n.3, p.652-659, 1980. Disponível em: <http://jas.fass.org/ cgi/content/abstract/51/3/652>. Acesso em: 14 set. 2010.

TSUTSUI, K. et al. A novel avian hypothalamic peptide inhibiting gonadotropin release. Biochemical and Biophysical Research Communications, v.275, n.2, p.661667, 2000. Disponível em: <http://www.ncbi.nlm.nih.gov/ e n trez/query.f c g i c m d = Retri eve \& d b $=$ P u b Med\&dopt $=$ Citation\&list_uids $=10964719>$. Acesso em: 14 set. 2010. doi: $10.1006 /$ bbrc. 2000.3350 .

UKENA, K. et al. Distribution of a novel avian gonadotropininhibitory hormone in the quail brain. Cell and Tissue Research, v.312, n.1, p.73-79, 2003. Disponível em: <http:/

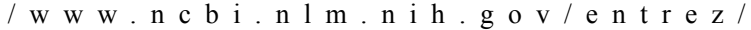
q u e r y. f c g i ? c m d $=R$ e t r i e v e $\&$ d b $=P$ u b Med\&dopt $=$ Citation\&list_uids $=12712318>$. Acesso em: 14 set. 2010. doi: $10.1007 / \mathrm{s} 00441-003-0700-x$.

WALTERS, D.L. et al. Pituitary and ovarian function in postpartum beef cows. II. Endocrine changes prior to ovulation in suckled and nonsuckled postpartum cows compared to cycling cows. Biology of Reproduction, v.26, n.4, p.647-654, 1982. Disponível em: <http://www.ncbi.nlm.nih.gov/entrez/ q u e r y. f c g i ? c m d $=\mathrm{R}$ e t r i e v e \& d b $=\mathrm{P} \mathrm{u} \mathrm{b}$ Med\&dopt $=$ Citation\&list_uids $=6282353>$. Acesso em: 14 set. 2010. doi: 10.1095/?biolreprod26.4.647.

WHISNANT, C.S. et al. Opioid inhibition of luteinizing hormone secretion during the postpartum period in suckled beef cows. Journal of Animal Science, v.63, n.5, p.14451448, 1986. Disponível em: <http://jas.fass.org/cgi/content/ abstract/63/5/1445>. Acesso em: 14 set. 2010.

WILLIAMS, G.L. et al. Mammary somatosensory pathways are not required for suckling-mediated inhibition of luteinizing hormone secretion and delay of ovulation in cows. Biology of Reproduction, v.49, n.6, p.1328-1337, 1993. Disponível em: <http:// www.ncbi.nlm.nih.gov/entrez/query.fcgi? $c m d=$ Retrieve $\& d b=P u b$ Med\&dopt=Citation\&list_uids=8286615>. Acesso em: 14 set. 2010. doi: 10.1095/?biolreprod49.6.1328.

YAMADA, S. et al. Inhibition of metastin (kisspeptin-54)GPR54 signaling in the arcuate nucleus-median eminence region during lactation in rats. Endocrinology, v.148, n.5, p.22262232, 2007. Disponível em: <http://www.ncbi.nlm.nih.gov/ entrez/query.f c g i ? c m d = Retrieve \& d b = P u b M ed\&dopt $=$ Citation\&list_uids $=17289848>$. Acesso em: 14 set. 2010. doi:10.1210/en.2006-1529.

ZHANG, Y. et al. Positional cloning of the mouse obese gene and its human homologue. Nature, v.372, n.6505, p.425432, 1994. Disponível em: <http://www.nature.com/nature/ journal/v372/n6505/abs/372425a0.html>. Acesso em: 14 set. 2010. doi:10.1038/372425a0. 